\title{
Senescence in cell oxidative status in two bird species with contrasting life expectancy
}

\author{
Pierre Bize $\cdot$ Sophie Cotting $\cdot$ Godefroy Devevey $\cdot$ \\ Juan van Rooyen · Fabrice Lalubin • Olivier Glaizot • \\ Philippe Christe
}

Received: 30 April 2013 / Accepted: 15 November 2013 / Published online: 30 November 2013

(C) Springer-Verlag Berlin Heidelberg 2013

\begin{abstract}
Oxidative stress occurs when the production of reactive oxygen species (ROS) by an organism exceeds its capacity to mitigate the damaging effects of the ROS. Consequently, oxidative stress hypotheses of ageing argue that a decline in fecundity and an increase in the likelihood of death with advancing age reported at the organism level are driven by gradual disruption of the oxidative balance at the cellular level. Here, we measured erythrocyte resistance to oxidative stress in the same individuals over several years in two freeliving bird species with contrasting life expectancy, the great tit (known maximum life expectancy is 15.4 years) and the Alpine swift (26 years). In both species, we found evidence for senescence in cell resistance to oxidative stress, with
\end{abstract}

Communicated by Oliver P Love.

Electronic supplementary material The online version of this article (doi:10.1007/s00442-013-2840-3) contains supplementary material, which is available to authorized users.

P. Bize $(\bowtie) \cdot$ S. Cotting $\cdot$ J. van Rooyen · F. Lalubin · O. Glaizot · P. Christe

Department of Ecology and Evolution, University of Lausanne, Biophore, 1015 Lausanne, Switzerland

e-mail: pierre.bize@unil.ch

Present Address:

P. Bize

Institute of Biological and Environmental Sciences, University of Aberdeen, Zoology Building, Tillydrone Avenue, AB24 2TZ, Aberdeen, UK

G. Devevey

Institute of Evolutionary Biology, University of Edinburgh,

King's Building, West Main Road, Edinburgh EH9 3JT, UK

O. Glaizot

Museum of Zoology, Place de la Riponne 6, 1014 Lausanne, Switzerland patterns of senescence becoming apparent as subjects get older. In the Alpine swift, there was also evidence for positive selection on cell resistance to oxidative stress, the more resistant subjects being longer lived. The present findings of inter-individual selection and intra-individual deterioration in cell oxidative status at old age in free-living animals support a role for oxidative stress in the ageing of wild animals.

Keywords Ageing - Antioxidant defences - Free radical theory of ageing $\cdot$ Life history theory $\cdot$ Oxidative stress

\section{Introduction}

The decline in fecundity and increase in likelihood of death with advancing age after an optimum, known as ageing or senescence, seems to affect virtually all organisms. Although ageing has been for a long time thought to be restricted to captive animals or humans (Masoro and Austad 2006), evidence is accumulating that ageing is also widespread in wild populations of vertebrates (Jones et al. 2008). Because ageing is a 'detrimental phenomenon', the mechanisms of ageing, the degree of their conservation among distant evolutionary lineages, and how such counter-selective phenomena persist in natural populations, remain the focus of intense research.

Nowadays, there is little doubt that ageing involves the failure of numerous control systems, which is well reflected by the plethora of mechanistic hypotheses of ageing reported in the biomedical literature, involving among other things disruption of the immune system (Helle et al. 2004) or cell membrane composition (Yu 2005). The vast majority of mechanistic hypotheses of ageing have, as common denominators, an intimate association between system failure and the accumulation of somatic damage. Because 
oxidative stress is a predominant cause of somatic damage, it has led to synthetic hypotheses pointing to oxidative stress, in a broad sense, as a major driver of ageing (Finkel and Holbrook 2000; Dowling and Simmons 2009; Selman et al. 2012; Sohal and Orr 2012). Oxidative stress hypotheses of ageing are organised around two paradoxes. The first one, best known as the free radical hypothesis of ageing, points out that despite mitochondrial respiration being vital as an energy source, it also produces damaging reactive oxygen species (ROS) as by-products (Harman 1956). ROS is thought to represent $0.1-4 \%$ of total oxygen consumed during normal respiration (Cadenas and Davies 2000), and the accumulation of ROS-induced damage on DNA, proteins, and lipids is thought to contribute to ageing (Beckman and Ames 1998; Finkel and Holbrook 2000). The second paradox, which has only emerged in the last decade, emphasizes that, although long-term exposure to ROS is debilitating, short and moderate peaks of ROS are vital on-off molecular signals via their action on the cyclic oxidation-reduction of cysteine residues present in kinases, phosphatases and other regulatory factors (Hamanaka and Chandel 2010). This second paradox illustrates that the relationship between oxidative stress and ageing is more complex than initially formulated in the free radical hypothesis of ageing (Ristow and Schmeisser 2011; Sohal and Orr 2012). Taken together, these hypotheses predict that, although oxidative stress occurs when the production of ROS exceeds the capacity of the antioxidant and repair machinery (Beckman and Ames 1998; Finkel and Holbrook 2000), ageing is likely to be dictated at the intra-cellular level by subtle changes in oxygen consumption, ROS production, antioxidant defences and repair mechanisms, with ageing being manifested at the cellular level by the deterioration of cell oxidative status with advancing age (Ristow and Schmeisser 2011; Sohal and Orr 2012). Hence, studies focusing on cell oxidative status, defined as the sum of processes influencing cell oxygen consumption, ROS production, antioxidant defences and repair mechanisms, might provide a suitable approach to address the importance of oxidative stress in shaping organismal ageing. Accordingly, there is growing evidence in humans and model organisms of the effect of advancing age on the disruption of cell oxidative status and on its etiological association with various diseases and morbidities such as cancer, cell degeneration, and inflammation (Valko et al. 2007). Whether the same processes occur in wild populations of vertebrates remain contentious.

In this study, we address the occurrence of age-related deterioration of cell oxidative status in two free-living bird species with contrasting life expectancy: the short-lived great tit (Parus major) and the long-lived Alpine swift (Apus melba), with respectively maximum expected life spans of 15.4 and 26 years in the wild (data from the web database AnAge; Tacutu et al. 2013). In both species, we gathered repeated measurements in the same individuals of erythrocyte resistance to an oxidative burst. Resistance of erythrocytes to oxidative stress is the sum of multiple factors, including membrane lipid composition, levels of intraand extra-cellular antioxidant defences (i.e. antioxidant compounds and enzymes) to past exposure of a cell membrane and antioxidant defences to oxidative stress (e.g. Lesgards et al. 2002; Rizzo et al. 2012). As such, erythrocytes provide valuable insight on cell oxidative status (Richards et al. 1998; Kiefer and Snyder 2000; Tsantes et al. 2006; Hattangadi and Lodish 2007), and avian erythrocytes might be especially interesting in this context because they contain functional mitochondria (Stier et al. 2013). Furthermore, previous studies in captive as well as wild birds have revealed a tight association between erythrocyte resistance to oxidative stress and fecundity or survival (Alonso-Alvarez et al. 2006; Bize et al. 2008; Losdat et al. 2013), indicating that this cellular biomarker can also provide valuable insight at the whole organism level. Because age-related decline in individual resistance to oxidative stress can be masked by the selective disappearance of the less resistant individuals (van de Pol and Verhulst 2006), we used a centring statistical approach where the age at last measurement (ALM) and chronological age centred on ALM were entered as two fixed terms in the same mixed model (van de Pol and Verhulst 2006; Reed et al. 2008). The first term allows testing for the selective disappearance of particular phenotype at the population level (i.e. changes in betweenindividual composition), and the second term for withinindividual changes in resistance to oxidative stress in the years of life preceding its last measurement (i.e. ageing per se) (van de Pol and Verhulst 2006; Reed et al. 2008). We also modelled the interaction between these two terms to test for the gradual appearance of senescence with advancing age.

\section{Materials and methods}

Study species and general procedures

The great tit is a $20 \mathrm{~g}$ non-migratory passerine that feeds on insects and seeds. This passerine inhabits forests and parks and nests in tree cavities or in artificial nest boxes. Female great tits usually lay one clutch of 5-12 eggs per year (modal clutch size is eight). Females incubate the clutch, and both parents feed the offspring up to fledging which occurs 20 days after hatching. Adult great tits are sexually mature at one year of age, they have a mean annual survival rate of ca. 0.47 (Payevsky 2006), and the oldest bird reported in our study population was 9 years old (PC; personal observation). Fieldwork was carried out in a great tit population nesting in artificial nest boxes located in woods in the vicinity of the 
University of Lausanne, Switzerland. This population has been monitored since 1989. A drop of blood was collected from nesting adults (2005-2010) and from their 12-days old nestlings in 2005 and 2007 (Christe et al. 2012).

The Alpine swift is a $90 \mathrm{~g}$ migrant colonial apodiform bird that breeds in holes in cliffs and under the roof of tall buildings. It feeds on insects caught exclusively in flight. It is socially monogamous and reproduces in colonies counting up to several hundred breeding pairs. A single clutch of 1-4 eggs is produced per year (modal clutch size is three). Males and females have equal investment in the brood. Both parents incubate the eggs for 20 days and feed the offspring up to fledging which takes place at 5069 days of age after hatching. Adult Alpine swifts are sexually mature at the age of two or 3 years (Tettamanti et al. 2012), they have a mean annual survival rate of ca. 0.77 (Bize et al. 2006), and the oldest recaptured bird reported in our population was 26 years old (Bize et al. 2009). Natal and breeding dispersal is limited with most birds breeding in the colony where they were born. Fieldwork was carried out in two Alpine swift colonies located under the roofs of tall buildings in Bienne and Solothurn, Switzerland, where nestlings have been ringed each year since at least 1968 and adults have been subjected to an individual based study since 2000 (Bize et al. 2008, 2009). We measured erythrocyte resistance to oxidative stress in 2005-2008 by collecting a drop of blood from adults, captured while sitting on eggs or provisioning their brood, and from 50-day-old nestlings.

Measurements of erythrocyte oxidative status

We assessed erythrocyte oxidative status as the time needed to hemolyse $50 \%$ of the cells exposed to a controlled ROS attack using the KRL bioassay (Brevet Spiral V02023; Kirial, Courernon, France). Immediately after the blood sampling, 10 or $16 \mu 1$ of blood was diluted in 365 or $584 \mu 1$ of KRL buffer adjusted to avian cell osmolarity in the great tit or in the Alpine swift, respectively. Samples were stored at $4{ }^{\circ} \mathrm{C}$ in the field before analysis in the laboratory, which occurred within $24 \mathrm{~h}$ after blood collection. Ninety microlitres of KRL-diluted blood was incubated at $40{ }^{\circ} \mathrm{C}$ and submitted to a controlled ROS attack by adding $153 \mu \mathrm{l}$ of a solution at $150 \mathrm{mmol}$ of 2,2'-azobis-(aminodinopropane) hydrochloride diluted in KRL buffer. The time needed to lyse $50 \%$ of the erythrocytes was assessed with a microplate reader device that follows the decrease of optical density at $540 \mathrm{~nm}$, thus allowing us to follow the cellular release of haemoglobins in each well. Samples were run in duplicates (mean $\pm \mathrm{SE}$ intra-plate coefficient of variation, $1.53 \% \pm 0.20$ in the great tit and $0.83 \% \pm 0.05$ in the Alpine swift), and mean sample values were calculated for the analyses.
Because changes in erythrocyte resistance to an oxidative burst can be influenced by changes in membrane lipid composition, in levels of antioxidant defences and in past exposure of cell membranes to oxidative stress (Brzezinska-Slebodzinska 2001; Lesgards et al. 2002; Rizzo et al. 2012), hereafter we refer to this measurement as 'erythrocyte oxidative status'.

\section{Ethical note}

This work was conducted under licences of the Veterinary Services of the Cantons Berne and Solothurn for the Alpine swift and of the Canton Vaud for the great tit. Ringing permits were provided by the Swiss Federal Agency for Environment, Forests and Landscapes.

\section{Data sets and statistical analyses}

To examine within-individual variation over age in resistance to oxidative stress, we restricted our analyses to subjects fulfilling the following conditions: (1) individuals were of known age (i.e. ringed as nestlings or defined as 1 year of age in adult tits based on plumage colouration); and (2) individuals were measured for resistance to oxidative stress over at least 2 different years. In total, we had 93 measurements in 33 great tit subjects and 734 measurements in 229 Alpine swift subjects. The range of ages across all the subjects varied between 12 days and 7 years of age (mean age at sampling $\pm \mathrm{SE}=2.79 \pm 0.18, n=93$ ) in the great tit and between 50 days and 22 years of age (mean age at sampling $\pm \mathrm{SE}=6.02 \pm 0.14, n=734$ ) in the Alpine swift.

We teased apart between- and within-individual changes in resistance to oxidative stress with advancing age using mixed models and a data centring approach (van de Pol and Verhulst 2006). That is, we modelled the effect of age in relation to ALM (i.e. age is centred on ALM), so that age 0 coincides with the last measurement, age -1 to 1 year before the last measurement, age -2 to 2 years before the last measurement (YBLM), etc.. This data centring is useful when focusing on age-related changes in the YBLM [see Reed et al. (2008) for a similar approach]. In the statistical models, we entered ALM, YBLM, and sex as three fixed terms, plus all possible interactions, and we entered individual identity and year of sampling as two random terms. For each individual, ALM defined the upper limit of the age window over which resistance to oxidative stress is studied, and YBLM allows the study of variation in resistance to oxidative stress in the years preceding the last measurement. Consequently, ALM allows testing for the selective contribution of some age window (and by extension phenotypes) to patterns of senescence in resistance to oxidative stress at the population level (i.e. between-individual 
effect), whereas YBLM allows testing for within-individual changes in resistance to oxidative stress (van de Pol and Verhulst 2006; Reed et al. 2008). We also entered the quadratic effects for the variables ALM and YBLM to gather information, respectively, on the shape of selection on ALM (e.g. directional vs. stabilizing) and on individual constancy in age-related variation in cell resistance to oxidative stress.

The interaction between YBLM and ALM allowed testing for gradual appearance of senescence with advancing age, or in other words for gradual changes in the slope of the relationship between age and cell resistance to oxidative stress. To help visualize and interpret this interaction between two continuous explanatory variables, we divided individuals into three ALM classes: young, middle-aged and old. Great tit subjects were 1-2, 3-5 and 6-7 years ALM in the young, middle-aged and old ALM classes, respectively. Alpine swift subjects were 1-3, 4-9 and $10+$ years ALM in the young, middle-aged and old ALM classes, respectively. Within each species, the young ALM class refers to individuals measured either before they started breeding or during their first reproductive attempt(s) (Harvey et al. 1979; Tettamanti et al. 2012), the middleaged class describes mature breeding adults, whereas the old age class presumably contains senescing individuals [following ageing patterns reported in Jones et al. (2008)]. We also re-ran mixed-models with YBLM and sex as two fixed terms, and with individual identity and year of sampling as two random terms to investigate significant agerelated changes in resistance to oxidative stress in these three age categories.

In the Alpine swift, ALM did not always coincide with the age at last appearance (and presumably death), since 136 of the 229 subjects were recaptured afterwards without record of resistance to oxidative stress (against one of the 33 great tit subjects). Hence, although a significant effect of ALM on resistance to oxidative stress would point toward selective disappearance of particular subjects, in the Alpine swift we also ran a parametric survival analysis with individual mean resistance to oxidative stress as an exploratory variable and age at last appearance based on individual re-sighting until 2012 as a response variable to formally address the influence of resistance to oxidative stress on life expectancy. When running survival analyses, 49 Alpine swift subjects still alive in 2012 were right censored and the survival distribution that best fitted the data was chosen based on Akaike information criterion. Furthermore, to formally address oxidative senescence in the years before last appearance, and presumably death, in both species we re-ran similar mixed models by restricting the data sets to subjects where ALM coincided with the presumed age at death (AD).

We have shown elsewhere that the time interval between blood sampling in the field and measurement of cell oxidative status in the laboratory can significantly influence cell resistance to oxidative stress $[P<0.05$; Bize et al. (2008)]. The inclusion of a time interval in the different analyses did not alter the present findings and, for shake of simplicity, we did not retain this covariate in our final statistical analyses.

All the statistical analyses were performed using the statistical package JMP version 8.0 (SAS Institute). Results are reported as mean \pm SE. Significant results are for $P<0.05$. Number of subjects $(N)$ and number of observations $(n)$ are reported whenever appropriate.

\section{Results}

Erythrocyte resistance to oxidative stress in the great tit and in the Alpine swift was significantly explained by the interaction between ALM and chronological YBLM (Table 1). In both species, this interaction is explained by the fact that patterns of within-individual changes in resistance to oxidative stress varied across age windows (Fig. 1). In the great tit, resistance to oxidative stress showed withinindividual improvement in the first years of life, the pattern being most apparent in middle-aged subjects, followed by senescence in resistance to oxidative stress in old-age subjects (Fig. 1a-c). There was no significant relationship between cell resistance to oxidative stress and YBLM in the young ALM class (effect of YBLM, $0.65 \pm 2.05$; $d f=12.6, t=0.32, P=0.76$ ), whereas this relationship was significantly positive in the middle-aged ALM class $(1.96 \pm 0.61 ; d f=32.9, t=3.19, P=0.003)$ and negative in the old ALM class $(-1.53 \pm 0.37 ; d f=19.1, t=-4.15$, $P<0.001)$. In the Alpine swift, resistance to oxidative stress showed slow but constant within-individual deterioration throughout life, with senescence being most apparent in old-age subjects (Fig. 1d-f). There was no significant relationship between cell resistance to oxidative stress and YBLM in the young ALM class $(0.84 \pm 0.59 ; d f=81.3$, $t=1.43, P=0.16$ ), and a significant negative relationship in the middle-aged ALM class $(-2.68 \pm 0.30 ; \mathrm{df}=438.5$, $t=-8.86, P<0.001)$ and old ALM class $(-2.94 \pm 0.55$; $d f=130.8, t=-5.29, P<0.001)$. We found no evidence of a quadratic effect of YBLM (all $P$ values $>0.30$ ), thus suggesting linear, constant, variation in cell resistance to oxidative status in the different age windows. Resistance to oxidative stress was not explained by the effect of sex, alone or in interaction with ALM or YBLM; non-significant interactions were dropped from the final model reported in Table 1. Additional analyses restricted to subjects where ALM coincided with the presumed AD show quantitatively similar results (Table S1), thus confirming the occurrence of oxidative senescence before death for individuals belonging mostly to old-age categories. 
Table 1 Results of generalised linear mixed models showing effects of sex, age at last measurement $(A L M)$, years before last measurement $(Y B L M)$, plus the interaction between ALM and YBLM, on resistance of red blood cell membranes to an oxidative burst measured in great tits and Alpine swifts

\begin{tabular}{|c|c|c|c|c|c|c|c|c|c|c|}
\hline & \multicolumn{5}{|c|}{ Great tit ( $N=33$ subjects, $n=93$ observations) } & \multicolumn{5}{|c|}{ Alpine swift ( $N=229$ subjects, $n=734$ observations) } \\
\hline & \multicolumn{5}{|c|}{ Random effects } & \multicolumn{5}{|c|}{ Random effects } \\
\hline & Var. comp. & \multicolumn{2}{|c|}{$95 \% \mathrm{CI}$} & \multicolumn{2}{|c|}{$\%$ Var. explained } & Var. comp. & \multicolumn{2}{|c|}{$95 \% \mathrm{CI}$} & \multicolumn{2}{|c|}{$\%$ Var. explained } \\
\hline Subject ID & 7.85 & \multicolumn{2}{|c|}{$(2.77-69.26)$} & \multicolumn{2}{|c|}{$21.9 \%$} & 3.84 & \multicolumn{2}{|c|}{$(2.43-10.34)$} & \multicolumn{2}{|c|}{$6.9 \%$} \\
\hline Year sampling & 0.16 & \multicolumn{2}{|c|}{$(0.44-)$} & \multicolumn{2}{|c|}{$0.5 \%$} & 25.53 & \multicolumn{2}{|c|}{$(7.82-368.11)$} & \multicolumn{2}{|c|}{$38.7 \%$} \\
\hline \multirow[t]{3}{*}{ Residual } & 27.72 & \multicolumn{2}{|c|}{$(19.28-43.24)$} & \multicolumn{2}{|c|}{$77.6 \%$} & 34.79 & \multicolumn{2}{|c|}{$(30.87-39.42)$} & \multicolumn{2}{|c|}{$54.4 \%$} \\
\hline & \multicolumn{5}{|c|}{ Fixed effects } & \multicolumn{5}{|l|}{ Fixed effects } \\
\hline & Estimate & SE & $d f$ & $t$ & $P$ & Estimate & SE & $d f$ & $t$ & $P$ \\
\hline ALM & 0.34 & 0.52 & 22.9 & 0.65 & 0.522 & 0.38 & 0.09 & 233.3 & 4.22 & $<0.001$ \\
\hline $\mathrm{ALM} \times \mathrm{ALM}$ & - & - & - & - & - & -0.04 & 0.01 & 234.7 & -2.74 & 0.007 \\
\hline YBLM & 1.16 & 0.63 & 6.5 & 1.85 & 0.110 & -0.19 & 0.36 & 320.0 & -0.55 & 0.584 \\
\hline $\mathrm{ALM} \times \mathrm{YBLM}$ & -0.96 & 0.30 & 72.8 & -3.23 & 0.002 & -0.16 & 0.06 & 665.9 & -2.51 & 0.012 \\
\hline $\operatorname{Sex}(F)$ & -0.92 & 0.81 & 24.1 & -1.14 & 0.267 & -0.45 & 0.26 & 210.0 & -1.74 & 0.083 \\
\hline Intercept & 77.69 & 2.02 & 23.0 & 38.39 & $<0.001$ & 53.76 & 2.58 & 3.3 & 20.87 & $<0.001$ \\
\hline
\end{tabular}

ALM squared $(A L M \times A L M)$ was retained only in the final model for the Alpine swift. Subject identity $(I D)$ and year of sampling were entered as random factors to control for multiple blood sampling of the same subjects. Denominator $d f$ were approximated by the Satterthwaite method

Var. Variance, $C I$ confidence interval
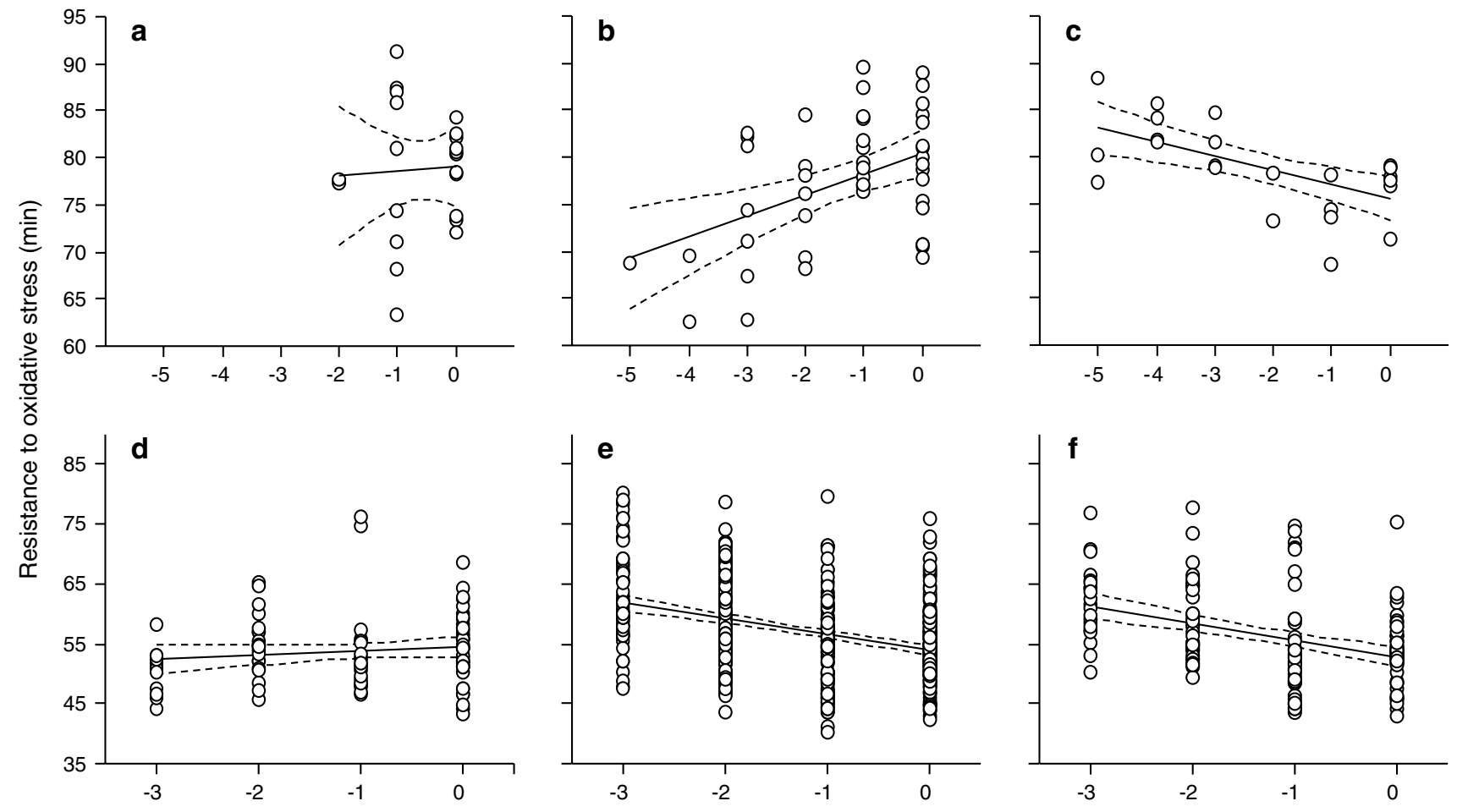

Years before last measurement $(\mathrm{yr})$

Fig. 1 Relationships between years before last measurement (YBLM) and resistance to oxidative stress in the short-lived great tit $(\mathbf{a}-\mathbf{c})$ and in the long-lived Alpine swift $(\mathbf{d}-\mathbf{f})$. For illustrative purpose, individuals were divided into three age at last measurement (ALM) classes: young (a, d), middle aged (b, e) and old (c, f). Great tit subjects were $1-2,3-5$ and 6-7 years ALM in the young, middle-aged and old ALM classes, respectively. Alpine swift subjects were 1-3, 4-9 and 10+ years ALM in the young, middle-aged and old ALM classes, respectively. Each dot is one observation per subject per YBLM class, and fitted lines are shown with $95 \%$ confidence intervals (dashed lines) 


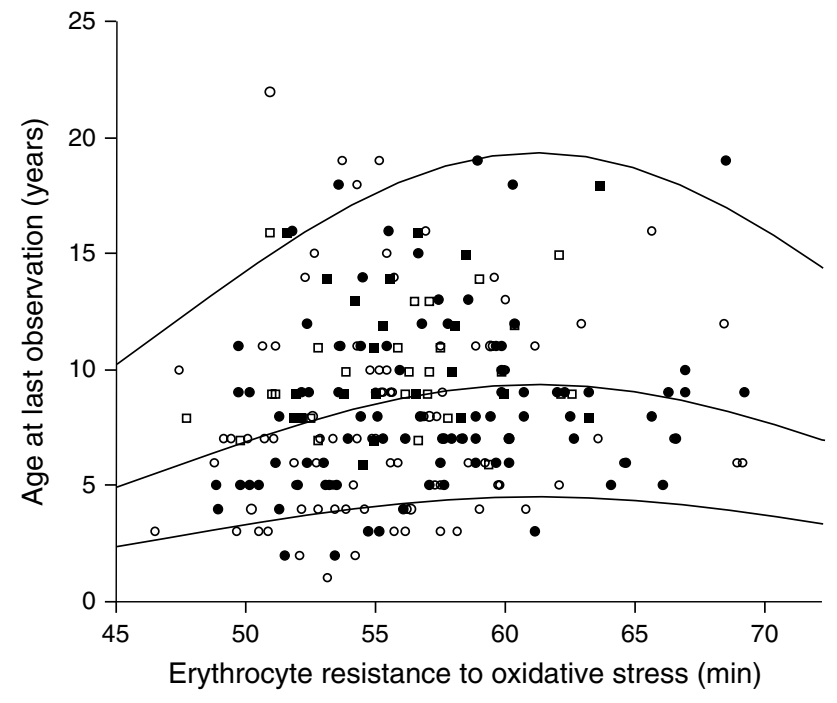

Fig. 2 Age at last observation, and by extrapolation life expectancy, in relation to erythrocyte mean residual resistance to oxidative stress in the long-lived Alpine swift. Individuals still alive in $2012(N=49)$ are shown as squares and those not re-sighted in $2012(N=180)$ as circles. Solid symbols male subjects, open symbols female subjects. Survival quantile lines $0.1,0.5$ and 0.9 are drawn

The significant and positive effect in Table 1 of ALM and ALM squared on resistance to oxidative stress in the Alpine swift $(0.35 \pm 0.09$ and $P<0.001$, and $-0.03 \pm 0.01$ and $P=0.011)$ hints at the existence of directional selection for more resistant phenotypes in this bird species, with the strength of selection flattening as individuals get older. This evidence is confirmed by fitting a lognormal survival analysis on age at last appearance based on individual re-sighting until 2012 in relation to mean individual resistance to oxidative stress. It shows that resistance to oxidative stress (fitted with a quadratic effect) was a significant predictor of survival in the Alpine swift (resistance to oxidative stress, $0.023 \pm 0.009, \chi^{2}=5.94, P=0.015$; resistance to oxidative stress squared, $-0.002 \pm 0.001, \chi^{2}=3.24, P=0.07$ ), with the existence of directional selection on low values of resistance to oxidative stress and a tendency for selection to flatten in individuals with high resistance to oxidative stress (Fig. 2). The effect of sex, alone or in interaction with resistance to oxidative stress, did not significantly explain the survival of Alpine swifts ( $P$ values $>0.41)$.

\section{Discussion}

The study of oxidative stress is challenging because, by definition, oxidative stress is the outcome of complex interactions between ROS production, antioxidant defences and repair mechanisms, and consequently there is no gold marker of oxidative stress (Monaghan et al. 2008; Selman et al. 2012). The study of ageing also has its own challenges, since it requires repeated sampling of the same individuals coupled with adequate statistical analyses to tease apart age-related changes due to demographic effects (i.e. selection) from within-individual effects (i.e. ageing per se) (van de Pol and Verhulst 2006). In the present study, we tried to take up these two challenges by analysing data sets in natural populations of the short-lived great tit and the long-lived Alpine swift for which we gathered repeated measures of erythrocyte resistance to oxidative stress of the same subjects. Our study emphasises in both species that cell oxidative status can deteriorate over age, with patterns of senescence becoming apparent in older subjects. In the Alpine swift, we also found evidence of positive selection on cell resistance to oxidative stress, the more resistant subjects being longer lived.

Erythrocytes, oxidative status and ageing

Variation in erythrocyte resistance to oxidative stress is doubtless the fruit of multiple, intertwined processes (Tsantes et al. 2006), and several non-exclusive mechanisms can be advanced to account for senescence in erythrocyte oxidative status, including changes in mitochondrial ROS production (Stier et al. 2013), in membrane lipid composition (Hulbert 2008), or in intracellular levels of antioxidant defences (Marinkovic et al. 2007). However, the relative contribution of these different processes in shaping variation in erythrocyte resistance to oxidative stress is currently unknown. Our measure of erythrocyte oxidative status is integrative, hitherto preventing us to decipher the exact mechanism(s) that triggered cell senescence in older individuals. To address this issue, studies are required where, ideally, all components of oxidative stress (i.e. ROS production, membrane composition and antioxidant defences) are measured simultaneously and repeatedly over the lifetime of the same individuals (Monaghan et al. 2008; Selman et al. 2012). It is worth realising, however, that many candidate molecular pathways are involved in oxidative stress and cellular ageing, and not all individuals from the same population might exhibit senescence in oxidative stress via dysfunction of the same pathways. Genetics and environmental factors, and gene by environment interactions, are thought to regulate resistance to oxidative stress (Finkel and Holbrook 2000). Therefore, extensive genetic diversity and environmental variation of wild populations might explain the difficulties in providing unambiguous demonstrations in oxidative senescence of specific molecular pathways in nature (Selman et al. 2012). In the present study, we made the best of a bad situation by focusing on an integrative measure of cell state (rather than on specific molecular pathways), allowing us to demonstrate senescence in cell oxidative status in older subjects in two 
phylogenetically distant wild bird species that greatly differ in their maximum lifespan. Our results shed light on the value of studying oxidative stress at the whole cell level, and we hope that this study will encourage more comparisons of oxidative stress at the cell level [across species but also across tissues (e.g. Röhme 1981)] in addition to comparisons at the sub-cellular levels. Our findings provide support to the idea that oxidative stress can play an important 'public' role in the ageing and life history trade-offs from humans to wild populations of vertebrates (Partridge and Gems 2002; Monaghan et al. 2008; Dowling and Simmons 2009; Selman et al. 2012).

Between- and within-individual variation in ageing

Age-related changes in erythrocyte resistance to oxidative stress observed at the population level (Supplementary Fig. 1) are the outcomes of selection over time for individuals with higher resistance, resulting in demographic effects or ageing per se (i.e. within-individual variation) (van de Pol and Verhulst 2006). Cross-sectional studies in free-living or semi-captive animals show a contrasting picture of oxidative senescence in the wild, with either no (e.g. Nussey et al. 2009; Lecomte et al. 2010; Saino et al. 2011) or quadratic (Bize et al. 2008; Alonso-Álvarez et al. 2010; Devevey et al. 2010) age-related changes in levels of diverse oxidative stress markers. These discrepancies might be partly explained by confounding effects of selection and ageing since age-related decline in individual resistance to oxidative stress can be masked by the selective disappearance of the less resistant individuals (van de Pol and Verhulst 2006). Accordingly, the use of repeated measurements coupled with mixed-model analyses where ALM, chronological age centred on ALM, plus their interaction, were entered as fixed explanatory variables (van de Pol and Verhulst 2006; Reed et al. 2008) emphasize the importance of considering these two processes within one analytical framework.

A first important result from these analyses is, as mentioned already, the significant interaction between ALM and chronological age centred on ALM on the whole dataset (Table 1), or on data restricted to subjects where the last measurement of resistance to oxidative stress matches with the year of their presumed death (Table S1). This gives strong evidence for progressive deterioration in cell resistance to oxidative stress in the years before death, with patterns of senescence becoming clearer in older subjects. Interestingly, in the great tit there was also evidence for within-individual improvement in erythrocyte resistance to oxidative stress at early ages, which is similar to previous longitudinal findings in the zebra finch (Taeniopygia guttata), another small, short-lived passerine (Alonso-Alvarez et al. 2006; but see Saino et al. 2011).
The factors accounting for an increase in cell resistance to oxidative stress early in life are unknown and need further investigation. Because of their stringent requirements for flight, birds have been proposed to maintain high levels of baseline physiological performance, and in turn fitness, throughout life (Ricklefs 2010), and their death has been defined as 'catastrophic' if it comes from an acute and sudden system failure that occurs regardless of age (Coulson and Fairweather 2001; Ricklefs 2010). There is nowadays fast-growing evidence from longitudinal studies showing that birds in natural populations can exhibit gradual (rather than catastrophic) decline in reproduction and survival (e.g. Jones et al. 2008; Reed et al. 2008; Rebke et al. 2010). Similar findings on physiological markers remain, however, scarce and often inconclusive since they are mostly based on cross-sectional studies (e.g. Lecomte et al. 2010; Saino et al. 2011; but see Angelier et al. 2007; Moe et al. 2009; Alonso-Álvarez et al. 2010). The present results provide two-fold evidence, showing that baseline physiological condition is indeed maintained until older ages (Figure S1) but that senescence in the baseline physiological condition becomes nonetheless apparent in very old (or long-lived) individuals (Fig. 1). Oxidative stress has been implicated in the cause of many diseases in humans and laboratory animals (Finkel and Holbrook 2000) but much remains to be done in order to identify the causes of death in natural populations and, by doing so, causally link oxidative stress to ageing.

A second important result is the significant positive relationship between ALM and cell resistance to oxidative stress in Alpine swifts, which demonstrates selective disappearance from the population of the less resistant subjects. It is further supported by survival analyses demonstrating that individuals possessing the more resistant erythrocytes against an exogenous oxidative attack were the longer lived. Although the relationship between ALM and cell resistance to oxidative stress was positive too in the great tit (effect of ALM, $0.34 \pm 0.52$ ), it was far from significant $(P=0.52$; Table 1$)$. This could be due to a lack of statistical power, since we have fewer data in the great tit than in the Alpine swift ( $N=33$ vs. 229 subjects). Alternatively, because the great tit is smaller (20 vs. $90 \mathrm{~g}$ ) and shorter lived [maximum life span, 15.4 vs. 26 years (Tacutu et al. 2013)] than the Alpine swift, we cannot exclude weaker survival selection on cell resistance to oxidative stress in the great tit. Previous studies performed in free-living birds covering a wide range of size and maximum longevity showed that erythrocyte resistance to oxidative stress predicted short-term survival in nestling great tits (Losdat et al. 2013) and adult male Alpine swifts [Bize et al. (2008); see also AlonsoAlvarez et al. (2006) for similar finding on captive zebra finches], plasma lipid peroxidation predicted recruitment 
in nestling European shags (Phalacrocorax aristotelis; $1,773 \mathrm{~g}$ and 30.6 years) (Noguera et al. 2012) and plasma antioxidant defences predicted long-term survival in adult barn swallows (Hirundo rustica; $18.3 \mathrm{~g}$ and 16 years) (Saino et al. 2011). Interestingly, the fact that erythrocytes are expendable, that they can penetrate the entire body via capillary distribution, and that the erythrocyte membrane is permeable to ROS which might allow the detoxification of extra-cellular ROS by the antioxidant defences in the erythrocyte cytosol, led to the hypothesis that erythrocytes can serve as an oxidative sink for the organism (Richards et al. 1998; Kiefer and Snyder 2000). Altogether, these results suggest that blood markers of oxidative stress provide important information on the intrinsic state and thereby, fitness of individuals in natural populations and in particular, that the role of erythrocyte oxidative status in shaping ageing patterns deserves further investigation (see also Patel et al. 2010).

Acknowledgments We are grateful to numerous students for their help in the field, to two anonymous reviewers for helpful comments, and to the Swiss National Science Foundation for financial support (grant no. 31003A_124988 to P. B. and 31003A_138187 to P. C.).

\section{References}

Alonso-Alvarez C, Bertrand S, Devevey G, Prost J, Faivre B, Chastel O, Sorci G (2006) An experimental manipulation of lifehistory trajectories and resistance to oxidative stress. Evolution 60:1913-1924

Alonso-Álvarez C, Pérez-Rodríguez L, García JT, Viñuela J, Mateo R (2010) Age and breeding effort as sources of individual variability in oxidative stress markers in a bird species. Physiol Biochem Zool 83:110-118

Angelier F, Weimerskirch H, Dano S, Chastel O (2007) Age, experience and reproductive performance in a long-lived bird: a hormonal perspective. Behav Ecol Sociobiol 61:611-621

Beckman KB, Ames BN (1998) The free radical theory of aging matures. Physiol Rev 78:547-581

Bize P, Gasparini J, Klopfenstein A, Altwegg R, Roulin A (2006) Melanin-based coloration is a nondirectionally selected sex-specific signal of offspring development in the Alpine swift. Evolution 60:2370-2380

Bize P, Devevey G, Monaghan P, Doligez B, Christe P (2008) Fecundity and survival in relation to resistance to oxidative stress in a free living bird. Ecology 89:2584-2593

Bize P, Criscuolo F, Metcalfe NB, Nasir L, Monaghan P (2009) Telomere dynamics rather than age predict life expectancy in the wild. Proc R Soc Lond B 276:1679-1683

Brzezinska-Slebodzinska E (2001) Erythrocyte osmotic fragility test as the measure of defence against free radicals in rabbits of different age. Acta Vet Hung 49:413-419

Cadenas E, Davies KJA (2000) Mitochondrial free radical generation, oxidative stress, and aging. Free Radic Biol Med 29:222-230

Christe P, Glaizot O, Strepparava N, Devevey G, Fumagalli L (2012) Twofold cost of reproduction: an increase in parental effort leads to higher malarial parasitaemia and to a decrease in resistance to oxidative stress. Proc R Soc Lond B 279:1142-1149
Coulson JC, Fairweather JA (2001) Reduced reproductive performance prior to death in the Black-legged Kittiwake: senescence or terminal illness? J Avian Biol 32:146-152

Devevey G, Bruyndonckx N, von Houwald F, Studer-Thiersch A, Christe P (2010) Age-specific variation of resistance to oxidative stress in the greater flamingo (Phoenicopterus ruber roseus). J Ornithol 151:251-254

Dowling DK, Simmons LW (2009) Reactive oxygen species as universal constraints in life-history evolution. Proc R Soc Lond B 276:1737-1745

Finkel T, Holbrook NJ (2000) Oxidants, oxidative stress and the biology of ageing. Nature 408:239-247

Hamanaka RB, Chandel NS (2010) Mitochondrial reactive oxygen species regulate cellular signaling and dictate biological outcomes. Trends Biochem Sci 35:505-513

Harman D (1956) Aging: a theory based on free radical and radiation chemistry. J Gerontol 11:208-300

Harvey PH, Greenwood PJ, Perrins CM, Martin AR (1979) Breeding success of great tits Parus major in relation to age of male and female parent. Ibis 121:216-219

Hattangadi SM, Lodish HF (2007) Regulation of erythrocyte lifespan: do reactive oxygen species set the clock? J Clin Invest 117:2075-2077

Helle S, Lummaa V, Jokela J (2004) Accelerated immunosenescence in preindustrial twin mothers. Proc Natl Acad Sci USA 101:12391-12396

Hulbert AJ (2008) The links between membrane composition, metabolic rate and lifespan. Comp. Biochem Physiol A Mol Int Physiol 150:196-203

Jones OR, Gaillard J-M, Tuljapurkar S, Alho JS, Armitage KB, Becker PH, Bize P, Brommer J, Charmantier A, Charpentier M, Clutton-Brock T, Dobson FS, Festa-Bianchet M, Gustafsson L, Jensen H, Jones CG, Lillandt B-G, McCleery R, Merilä J, Neuhaus P, Nicoll MAC, Norris K, Oli MK, Pemberton J, Pietiäinen H, Ringsby TH, Roulin A, Saether B-E, Setchell JM, Sheldon BC, Thompson PM, Weimerskirch H, Wickings EJ, Coulson T (2008) Senescence rates are determined by ranking on the fastslow life-history continuum. Ecol Lett 11:664-673

Kiefer CR, Snyder LM (2000) Oxidation and erythrocyte senescence. Curr Opin Hematol 72:113-116

Lecomte VJ, Sorci G, Cornet S, Jaeger A, Faivre B, Arnoux E, Gaillard M, Trouvé C, Besson D, Chastel O, Weimerskirch H (2010) Patterns of aging in the long-lived wandering albatross. Proc Natl Acad Sci USA 107:6370-6375

Lesgards JF, Durand P, Lassarre M, Stocker P, Lesgards G, Lanteaume A, Prost M, Lehucher-Michel MP (2002) Assessment of lifestyle effects on the overall antioxidant capacity of healthy subjects. Environ Health Perspect 110:479-486

Losdat S, Helfenstein F, Blount JD, Marri V, Maronde L, Richner H (2013) Nestling erythrocyte resistance to oxidative stress predicts fledging success but not local recruitment in a wild bird. Biol Lett $13: 1$

Marinkovic D, Zhang X, Yalcin S, Luciano JP, Brugnara C, Huber T, Ghaffari S (2007) Foxo3 is required for the regulation of oxidative stress in erythropoiesis. J Clin Invest 117:2133-2144

Masoro EJ, Austad SN (2006) Handbook of the biology of aging. Academic Press, Burlington

Moe B, Rønning B, Verhulst S, Bech C (2009) Metabolic ageing in individual zebra finches. Biol Lett 5:86-89

Monaghan P, Metcalfe NB, Torres R (2008) Oxidative stress as a mediator of life history trade-offs: mechanisms, measurements and interpretation. Ecol Lett 12:75-92

Noguera JC, Kim S-Y, Velando A (2012) Pre-fledgling oxidative damage predicts recruitment in a long-lived bird. Biol Lett 8:61-63 
Nussey DH, Pemberton JM, Pilkington JG, Blount JD (2009) Life history correlates of oxidative damage in a free-living mammal population. Funct Ecol 23:809-817

Partridge L, Gems D (2002) Mechanisms of ageing: public or private? Nat Rev Gen 3:165-175

Patel KV, Semba RD, Ferrucci L, Newman AB, Fried LP, Wallace RB, Bandinelli S, Phillips CS, Yu B, Connelly S, Shlipak MG, Chaves PHM, Launer LJ, Ershler WB, Harris TB, Longo DL, Guralnik JM (2010) Red cell distribution width and mortality in older adults: a meta-analysis. J Gerontol A Biol Sci Med Sci 65A:258-265

Payevsky VA (2006) Mortality rate and population density regulation in the great tit, Parus major: a review. Russ J Ecol 37:180-187

Rebke M, Coulson T, Becker PH, Vaupel JW (2010) Reproductive improvement and senescence in a long-lived bird. Proc Natl Acad Sci USA 107:7841-7846

Reed TE, Kruuk LEB, Wanless S, Frederiksen M, Cunningham EJA, Harris MP (2008) Reproductive senescence in a long-lived seabird: rates of decline in late-life performance are associated with varying costs of early reproduction. Am Nat 171:E89-E101

Richards RS, Roberts TK, McGregor NR, Dunstan RH, Butt HL (1998) The role of erythrocytes in the inactivation of free radicals. Med Hypoth 50:363-367

Ricklefs RE (2010) Insights from comparative analyses of aging in birds and mammals. Aging Cell 9:273-284

Ristow M, Schmeisser S (2011) Extending life span by increasing oxidative stress. Free Rad Biol Med 51:327-336

Rizzo AM, Corsetto PA, Montorfano G, Milani S, Zava S, Tavella S, Cancedda R, Berra B (2012) Effects of long-term space flight on erythrocytes and oxidative stress of rodents. PLoS ONE 7:e32361

Röhme D (1981) Evidence for a relationship between longevity of mammalian species and life spans of normal fibroblasts in vitro and erythrocytes in vivo. Proc Natl Acad Sci USA 78:5009-5013
Saino N, Caprioli M, Romano M, Boncoraglio G, Rubolini D, Ambrosini R, Bonisoli-Alquati A, Romano A (2011) Antioxidant defenses predict long-term survival in a passerine bird. PLoS ONE 6

Selman C, Blount JD, Nussey DH, Speakman JR (2012) Oxidative damage, ageing, and life-history evolution: where now? Trends Ecol Evol 27:570-577

Sohal RS, Orr WC (2012) The redox stress hypothesis of aging. Free Rad Biol Med 52:539-555

Stier A, Bize P, Schull Q, Zoll J, Singh F, Geny B, Gros F, Royer C, Massemin S, Criscuolo F (2013) Avian erythrocytes have functional mitochondria, opening novel perspectives for birds as animal models in the study of ageing. Front Zool 10:33

Tacutu R, Craig T, Budovsky A, Wuttke D, Lehmann G, Taranukha D, Costa J, Fraifeld VE, de Magalhaes JP (2013) Human ageing genomic resources: integrated databases and tools for the biology and genetics of ageing. Nucleic Acids Res 41:D1027-D1033

Tettamanti F, Witvliet W, Bize P (2012) Selection on age at first and at last reproduction in the long-lived Alpine Swift Apus melba. Ibis 154:338-344

Tsantes AE, Bonovas S, Travlou A, Sitaras NM (2006) Redox imbalance, macrocytosis, and RBC homeostasis Antiox. Redox Sign $8: 1205-1216$

Valko M, Leibfritz D, Moncol J, Cronin MTD, Mazur M, Telser J (2007) Free radicals and antioxidants in normal physiological functions and human disease. Int J Biochem Cell Biol 39:44-84

van de Pol M, Verhulst S (2006) Age-dependent traits: a new statistical model to separate within- and between-individual effects. Am Nat 167:766-773

Yu BP (2005) Membrane alteration as a basis of aging and the protective effects of calorie restriction. Mech Ageing Develop 126:1003 